\title{
Simple approximations for the pseudo-Jahn-Teller Hamiltonian using the coupled cluster method
}

\author{
Document Version \\ Accepted author manuscript
}

Link to publication record in Manchester Research Explorer

\section{Citation for published version (APA):}

Bishop, RF., Carter (Quick), RM., Davidson, NJ., \& van der Walt, DM. (2000). Simple approximations for the pseudo-Jahn-Teller Hamiltonian using the coupled cluster method. In R. F. Bishop, KA. Gernoth, NR. Walet, \& Y. Xian (Eds.), Recent Progress in Many-Body Theories: Proceedings of the 10th International Conference, Seattle, USA, September 10-15, 1999 (pp. 449-452). (Series on Advances in Quantum Many-Body Theory; Vol. 3). World Scientific Publishing Co. Pte. Ltd. http://www.worldscibooks.com/physics/4434.html

\section{Published in:}

Recent Progress in Many-Body Theories

\section{Citing this paper}

Please note that where the full-text provided on Manchester Research Explorer is the Author Accepted Manuscript or Proof version this may differ from the final Published version. If citing, it is advised that you check and use the publisher's definitive version.

\section{General rights}

Copyright and moral rights for the publications made accessible in the Research Explorer are retained by the authors and/or other copyright owners and it is a condition of accessing publications that users recognise and abide by the legal requirements associated with these rights.

\section{Takedown policy}

If you believe that this document breaches copyright please refer to the University of Manchester's Takedown Procedures [http://man.ac.uk/04Y6Bo] or contact uml.scholarlycommunications@manchester.ac.uk providing relevant details, so we can investigate your claim.

\section{OPEN ACCESS}




\section{Series on Advances in Quantum Many-Body Theory - Vol. 3}

The Proceedings of the 10th International Conference

\section{RECENT PROGRESS IN MANY-BODY THEORIES}

Seattle, USA September $10-15,1999$

Editors

Raymond F. Bishop

Klaus A. Gernoth

Niels R. Walet

Yang Xian

UMIST, Manchester, UK 


\section{Published by}

World Scientific Publishing Co. Pte. Ltd.

P O Box 128, Farner Road, Singapore 912805

USA office: Suite 1B, 1060 Main Street, River Edge, NJ 07661

UK office: 57 Shelton Street, Covent Garden, London WC2H 9HE

British Library Cataloguing-in-Publiçation Data

A catalogue record for this book is available from the British Library.

\section{RECENT PROGRESS IN MANY-BODY THEORIES (RPMBT-10)}

Copyright $\odot 2000$ by World Scientific Publishing Co. Pte. Ltd.

All rights reserved. This book, or parts thereof, may not be reproduced in any form or by any means, electronic or mechanical, including photocopying, recording or any information storage and retrieval system now known or to be invented, without written permission from the Publisher.

For photocopying of material in this volume, please pay a copying fee through the Copyright Clearance Center, Inc., 222 Rosewood Drive, Danvers, MA 01923, USA. In this case permission to photocopy is not required from the publisher.

ISBN 981-02-4318-9

Printed in Singapore by Uto-Print 


\title{
SIMPLE APPROXIMATIONS FOR THE PSEUDO-JAHN-TELLER HAMILTONIAN USING THE COUPLED CLUSTER METHOD
}

\author{
RAYMOND F. BISHOP \\ Department of Physics, UMIST, P.O. Box 88, Manchester M60 1QD, UK \\ RACHEL M. CARTER (QUICK), NEIL J. DAVIDSON \\ Department of Physics, University of Pretoria, Pretoria, 0002, South Africa \\ E-mail: rcarter@scientia.up.ac.za \\ DAVID M. VAN DER WALT \\ Department of Physics, Vista University, Mamelodi, South Africa
}

The normal coupled cluster method has been used to obtain simple accurate approximations to the ground and first excited state energies of the linear $e \otimes E$ pseudo-Jahn-Teller Hamiltonian.

\section{Introduction}

We consider a particularly simple, yet non-trivial, many-particle Hamiltonian, namely the linear $e \otimes E$ pseudo-Jahn-Teller (JT) Hamiltonian. This Hamiltonian, which describes the coupling between two degenerate bosonic modes and a two-level fermionic system, is given by

$$
\hat{H}=\frac{1}{2} \omega_{0} \sigma^{z}+\omega b_{1}^{\dagger} b_{1}+\omega b_{2}^{\dagger} b_{2}+\eta\left(b_{1}^{\dagger}+b_{1}\right) \sigma^{x}-\eta\left(b_{2}^{\dagger}+b_{2}\right) \sigma^{y} .
$$

Here $\omega$ denotes the angular frequency of the bosonic modes; $b_{1}^{\dagger}$ and $b_{2}^{\dagger}$ denote bosonic creation operators $\left[b_{1}, b_{1}^{\dagger}\right]=\left[b_{2}, b_{2}^{\dagger}\right]=1 ; \omega_{0}$ denotes the fermionic level splitting, $\sigma^{x}, \sigma^{y}$ and $\sigma^{z}$ are the Pauli matrices, and $\eta$ is the coupling strength. Clearly such a generic boson-fermion Hamiltonian is widely applicable. In particular it is extensively used in quantum chemistry, where it describes the vibronic coupling between two vibrational modes of a molecule/crystal and two (nearly) degenerate electronic levels $\left(\omega_{0} \ll \omega\right)$.

This Hamiltonian is also often written in terms of the circularly polarized modes,

$$
a_{1} \equiv \frac{1}{\sqrt{2}}\left(b_{1}+i b_{2}\right) \quad a_{2} \equiv \frac{1}{\sqrt{2}}\left(b_{1}-i b_{2}\right),
$$

of positive and negative helicity respectively as,

$$
\hat{H}=\frac{1}{2} \omega_{0} \sigma^{z}+\omega a_{1}^{\dagger} a_{1}+\omega a_{2}^{\dagger} a_{2}+\gamma\left(a_{1}+a_{2}^{\dagger}\right) \sigma^{+}+\gamma\left(a_{1}^{\dagger}+a_{2}\right) \sigma^{-}, \quad \gamma \equiv \eta / \sqrt{2} .
$$

Although this Hamiltonian is remarkably simple, no exact solution has yet been found. ${ }^{1 a}$ Analytical values for these energies are however available for isolated values of the coupling $\eta$ and/or the level splitting $\omega_{0}$. These are the so-called Juddian

\footnotetext{
${ }^{a}$ Excellent, yet not exact, results can be obtained using an expansion in terms of generalized spheroidal wave functions
} 
solutions, ${ }^{1}$ which have been used here to test the accuracy of the numerical results obtained from a large-scale diagonalization.

One also notes that this Hamiltonian has two symmetries, which should be respected by any approximation. The first is the parity symmetry which says that the eigenstates have either an even or an odd number of "excitation" quanta. The corresponding number operator is given by

$$
\hat{N} \equiv b_{1}^{\dagger} b_{1}+b_{2}^{\dagger} b_{2}+\frac{1}{2}\left[\sigma^{z}+1\right],
$$

and the parity operator is defined in terms of it as

$$
\hat{\Pi} \equiv \exp [i \pi \hat{N}] \text {. }
$$

The ground state has even and the first excited state odd parity. The second symmetry is angular momentum. The total angular momentum,

$$
\hat{J} \equiv \hat{L}_{z}+\hat{S}_{z}=i\left(b_{1}^{\dagger} b_{2}-b_{1} b_{2}^{\dagger}\right)+\frac{1}{2} \sigma^{z},
$$

is a constant of the motion with eigenvalues $j= \pm \frac{1}{2}, \pm \frac{3}{2} \cdots$. The ground state has $j=-\frac{1}{2}$ and the first excited state has $j=\frac{1}{2}$.

As a first step, only the ground-state and first excited-state energies for this Hamiltonian have been considered, and simple yet accurate approximations have been found using the normal coupled cluster method. ${ }^{2}$

\section{The coupled cluster method}

The coupled cluster method (CCM) is a very popular and successful ab initio manybody method. It has been widely applied in fields as diverse as quantum chemistry, nuclear physics, the electron gas, lattice gauge and continuum field theories, and spin and electron lattice models.

The basic idea behind the method is the $\exp (\hat{S})$ parameterization of the groundstate ket,

$$
|\psi\rangle=\mathrm{e}^{\hat{s}}|\phi\rangle,
$$

where $\hat{S}$ is the correlation operator, and $|\phi\rangle$ is the model or reference state, for which $\langle\phi \mid \psi\rangle \neq 0$. The correlation operator is then expanded as

$$
\hat{S}=\sum_{I} s_{I} C_{I}^{\dagger}
$$

where the set $\left\{C_{I}^{\dagger}\right\}$ form a complete commuting set of creation operators wrt $|\phi\rangle$, chosen for convenience such that $C_{I}|\phi\rangle=0=\langle\phi| C_{I}^{\dagger}$. The Schrödinger equation,

$$
\begin{aligned}
\hat{H}|\psi\rangle & =E_{0}|\psi\rangle \\
\Rightarrow \mathrm{e}^{-\hat{s}} \hat{H} \mathrm{e}^{\hat{s}}|\phi\rangle & =E_{0}|\phi\rangle,
\end{aligned}
$$


is then rewritten in terms of the similarity (not unitary) transformed Hamiltonian

$$
\mathrm{e}^{-\hat{S}} \hat{H} \mathrm{e}^{\hat{S}}=\hat{H}+[\hat{H}, \hat{S}]+\frac{1}{2 !}[[\hat{H}, \hat{S}], \hat{S}]+\cdots .
$$

In the normal coupled cluster method (NCCM) one projects the Schrödinger equation (9) onto $|\phi\rangle$ and $C_{I}^{\dagger}|\phi\rangle$ to give equations for the energy $E_{0}$ and the coefficients $\left\{S_{I}\right\}$ respectively,

$$
\begin{aligned}
\left\langle\phi\left|\mathrm{e}^{-\hat{S}} \hat{H} \mathrm{e}^{\hat{s}}\right| \phi\right\rangle & =E_{0}, \\
\left\langle\phi\left|C_{I} \mathrm{e}^{-\hat{S}} \hat{H} \mathrm{e}^{\hat{S}}\right| \phi\right\rangle & =0, \quad \forall I .
\end{aligned}
$$

These equations are of course exact. In practice however one must truncate the infinite sum over $I$ to a finite sum of order $N$ to obtain the so-called SUB- $N$ approximation.

The advantages of the method include its size extensivity (cluster property), the tractability of the similarity transform and the fact that the Feynman-Hellmann theorem is satisfied to all orders. There are however some disadvantages. To any finite order the method is manifestly non-Hermitian and the CCM energy may become complex. One has no lower bound property for the CCM energy and non-uniform convergence may occur. Lastly, although the correlation operator $\hat{S}$ always exists, a convergent expansion of the form (8) in terms of the complete set of creation operators may not exist. ${ }^{2}$

\section{Results from the CCM for the pseudo-Jahn-Teller Hamiltonian}

.. Introducing the quasi-boson operator

$$
c^{\dagger} \equiv b_{1}^{\dagger} \sigma^{x}-b_{2}^{\dagger} \sigma^{y}=\frac{1}{2}\left(a_{2}^{\dagger} \sigma^{+}+a_{1}^{\dagger} \sigma^{-}\right),
$$

which commutes with $\hat{J}$ and $\hat{\Pi}$, one can show that the ground (first-excited) state can be expanded as a power series in $c^{\dagger}$ acting on the state $|0\rangle|0\rangle|\downarrow\rangle(|0\rangle|0\rangle|\uparrow\rangle)$, where $|0\rangle|0\rangle$ is the bosonic vaccum, and that the equations for the first excited state are obtained from those for the ground state by the formal replacement $\omega_{0} \rightarrow-\omega_{0}$.

In practice the reference state for the CCM must give the zero coupling $\eta=0$ and infinite coupling $\eta \rightarrow \infty$ limits of the exact eigenstate. This avoids problems with the existence of a meaningful finite order $\exp (\hat{S})$ expansion. In this case the exact ground state of the resonant $\left(\omega_{0}=\omega\right)$ pseudo-Jahn-Teller Hamiltonian ${ }^{1}$ has been used as the CCM reference state, namely,

$$
|\phi\rangle=\left[I_{0}\left(2 \frac{\gamma^{2}}{\omega^{2}}\right)+I_{1}\left(2 \frac{\gamma^{2}}{\omega^{2}}\right)\right]^{-\frac{1}{2}}\left[I_{0}\left(2 \frac{\gamma}{\omega} c^{\dagger}\right)-I_{1}\left(2 \frac{\gamma}{\omega} c^{\dagger}\right)\right]|0\rangle|0\rangle|\downarrow\rangle,
$$

where $I_{0}$ and $I_{1}$ are modified Bessel functions of the first kind. Only a single correlation operator,

$$
\hat{S}=\dot{s}_{1}\left(c^{\dagger}+\frac{\gamma}{\omega}\right)
$$

has been used. One thus obtains only a single transcendental equation for $s_{1}$ from (12) and the energy from (11). The equations for the first excited state are obtained by replacing $\omega_{0}$ by $-\omega_{0}$. 

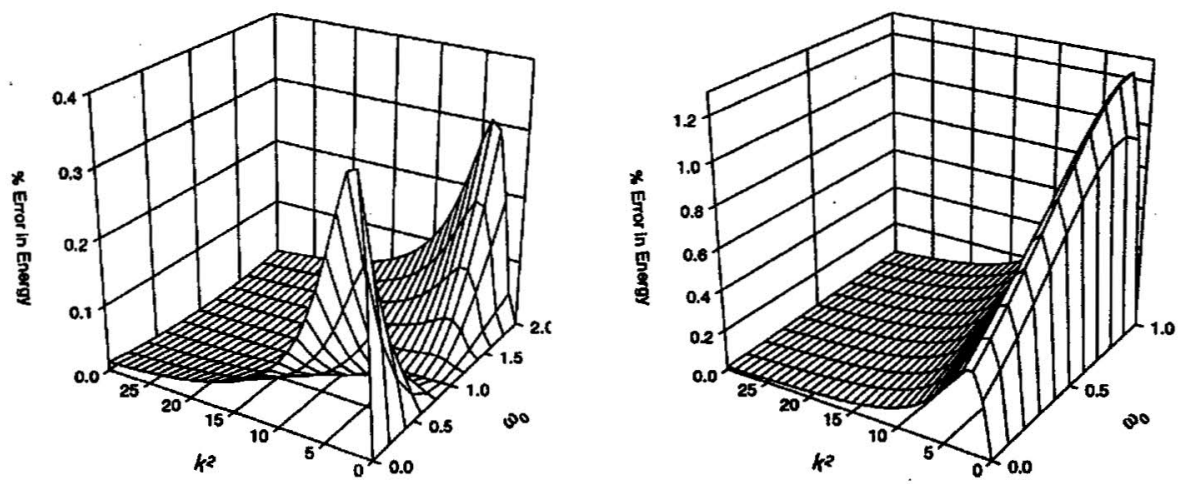

Figure 1. The percentage error in, the ground-state and first excited-state energies of the linear $e \otimes E$ pseudo-JT Hamiltonian obtained from first order NCCM calculations as a function of the coupling $k^{2}=2 \eta^{2}$ and the level splitting $\omega_{0}$.

\section{Conclusions and outlook}

As is evident in Fig. 1, the results are excellent. For the JT case $\left(\omega_{0}=0\right)$, the results are better than results from the CCM when applied to a unitary transform of the Hamiltonian, variational methods and other unitary-transformed methods. ${ }^{3}$ In particular for the ground-state energy the maximal error (when compared to the essentially exact numerical results) is only $0.4 \%$. For the first excited-state energy the maximal error is only $1.2 \%$.

Future work could involve the study of the time and temperature dependence of this Hamiltonian, and the study of related models such as the multi-mode Rabi Hamiltonian, the polaron problem and the $\Gamma_{8} \otimes \tau_{2}$ Hamiltonian.

\section{Acknowledgments}

R.F.B. acknowledges financial support from the Engineering and Physical Sciences Research Council (EPSRC) of UK. N.J.D., R.M.C., and D.M.vdW. acknowledge financial support from the National Research Foundation (NRF) of South Africa.

\section{References}

1. H. G. Reik et al., J. Phys. A 15, 3491 (1982); J. Phys. A 20, 6327 (1987); J. Phys. A 26, 6549 (1993);

2. R. F. Bishop et al., preprint; to appear in Condensed Matter Theories Vol. 15.

3. W. H. Wong, and C. F. Lo, Phys. Rev. B 54, 12859 (1996); Phys. Lett. A 223, 123 (1996). 